\title{
Novel Stability Criteria for T-S Fuzzy Systems
}

\author{
Xudong Zhao, Lixian Zhang, Member, IEEE, Peng Shi, Senior Member, IEEE, \\ and Hamid Reza Karimi, Senior Member, IEEE
}

$\mathbf{M}$ OST physical systems and processes in the real world are mathematically modeled in complex nonlinear systems, which causes difficulties when it comes to system analysis and synthesis. Among the available techniques used to model, analyze, and design, the well-known Takagi-Sugeno (T-S) fuzzy model approach has been recognized as being very effective at describing the complex nonlinear systems in a general framework [2], [4], [13], [45]. The T-S fuzzy models are described by a set of fuzzy "If . . . then" rules with fuzzy sets in the antecedents and linear time-invariant dynamic systems in the consequent, which can approximate many typical nonlinear systems.

So far, the T-S fuzzy model has been extensively applied in many dynamical systems, such as stochastic systems [21], neu-

Manuscript received July 18, 2012; revised October 21, 2012 and January 18 , 2013; accepted March 11, 2013. Date of publication March 22, 2013; date of current version March 27, 2014. This work was supported in part by the National Natural Science Foundation of China under Grant 61203123, Grant 60904001, and Grant 61174058, in part by the National Key Basic Research Program (973, No. 2012CB215202), in part by the 111 Project (B12018), in part by the Doctoral Fund of Ministry of Education of China under Grant 20092302120071, in part by the Fundamental Research Funds for the Central Universities, China, under Grant 11CX04044A, and in part by the Shandong Provincial Natural Science Foundation, China, under Grant ZR2012FQ019.

$\mathrm{X}$. Zhao is with the College of Information Science and Technology, Bohai University, Jinzhou 121013, China, and also with the College of Information and Control Engineering, China University of Petroleum, Qingdao 266555, China (e-mail: xdzhaohit@gmail.com).

L. Zhang is with the School of Astronautics, Harbin Institute of Technology, Harbin, 150001, China (e-mail: lixianzhang@ hit.edu.cn).

P. Shi was with the Department of Computing and Mathematical Sciences, University of Glamorgan, Pontypridd CF37 1DL, U.K. He is now with the School of Electrical and Electronic Engineering, The University of Adelaide, S.A. 5005, Australia (e-mail: peng.shi@vu.edu.au).

H. R. Karimi is with the Department of Engineering, Faculty of Technology and Science, University of Agder, Grimstad 4898, Norway (e-mail: hamid.r.karimi@uia.no).

Color versions of one or more of the figures in this paper are available online. ral networks [17], [22], switched systems [47], network control systems [14], etc. Therefore, many control issues of T-S fuzzy systems have been investigated, such as the fundamental problems of stability analysis and stabilization [6], [23], [36], reliable control strategies [44], $H_{\infty}$ control design [1], and fault detection and fault estimation observer design [15], [29], [46]. As a major concern and fundamental problem in the area, stability analysis has, to date, been extensively studied whereas most of results are obtained via quadratic Lyapunov function approach. It is also noted that a linear matrix inequality (LMI)based formulation of the stability problem is preferred in the literature, since the inherent flexibility of the LMI conditions facilitates the investigation of control synthesis problems, for T-S fuzzy systems [28]. In these methods, a numerical solution is efficiently obtained by convex optimization techniques such as the interior point method. Although LMI-based approaches have been successful, there is still a serious problem in that the results obtained via LMIs are quite conservative. Therefore, studies on less conservative criteria have been widely conducted, and some improvements have been made on the basis of various approaches, such as different types of Lyapunov function [4], [42], [43]. Generally speaking, most existing stability conditions of T-S fuzzy systems can be divided into two categories: membership-independent conditions and membershipdependent conditions.

For the derivations of membership-independent stability criteria for T-S fuzzy systems, the common Lyapunov function approach is naturally adopted; see, for example, [42], and further relaxed conditions are obtained in [41]. However, to find a common matrix that satisfies all Lyapunov inequalities is rather conservative, if not impossible in practice. Hence, several other methods have been developed to relax those stability conditions, among which, piecewise Lyapunov function [16] and fuzzy Lyapunov function [38] (different nomenclatures in the literature, such as nonquadratic Lyapunov function [4], basis-dependent Lyapunov function [48], and weighting dependent Lyapunov function [43]) have been well recognized to be effective. Using fuzzy Lyapunov functions, membership-independent conditions have been proposed in [4]. The fuzzy Lyapunov function, unlike piecewise Lyapunov function approach, consists of a fuzzy blending of multiple Lyapunov functions, which is therefore smooth and can avoid the nonconvex problems in the controller design. However, the serious drawback of the fuzzy Lyapunov function approach is that the time derivatives of membership functions will appear in the stability analysis [32]. To overcome this problem, a line-integral Lyapunov function is proposed in [32], where the Lyapunov function is formulated to be a line integral of a fuzzy vector along a path from the origin to the current state. Then, by introducing slack matrix variables into the LMI conditions of [32], less conservative 
membership-independent conditions are obtained in [25]. Other extensions of [32] can be found in [11] and references therein. Recently, by employing a polynomial Lyapunov function (but only in system state $x(t)$ ), new membership-independent conditions in sum of squares (SOS) formulation are proposed in [39] and [40], and it is interesting that the conservativeness of their conditions can be reduced with the growth of the degree of $x(t)$ in the constructed Lyapunov function.

On the other hand, it is noted that all the stability conditions in the literature mentioned previously are membershipindependent and therefore conservative to different extents, since those conditions must be fulfilled for an arbitrary membership function. In fact, the region of attraction for fuzzy systems may be membership-dependent [18], [19], [30], [35], i.e., if some information of the membership functions in a region around the equilibrium is known, relaxed stability conditions may be derived. Therefore, much more attention has been devoted to obtain membership-dependent stability conditions (see [3], [20] and references therein). For pioneer works in this area, see the results that are developed by common quadratic Lyapunov functions in [34] and references therein, where the knowledge of membership functions' shape is taken into account. In [38], by defining a fuzzy Lyapunov function approach, improved stability criteria that depend explicitly on the upper bound of time derivative of membership functions are established. In the light of a null product item, results of [38] have been generalized in [26]. By utilizing the appropriate properties of the time derivatives of membership functions, further improved stability conditions with different conservativeness have been presented in [9] and [25]. Broadly speaking, these results obtained by fuzzy Lyapunov functions are less conservative than those ignoring the information of the membership functions. In view of this, a novel type of Lyapunov function is explored in [24] and [27], where the function is given by a double parametrization. One is parameterized by the membership functions and the other is parameterized by the time derivatives of the membership functions. By this setting, the information that concerns the second time derivative of the membership functions becomes available to describe the time-varying characteristic of T-S fuzzy systems.

Therefore, properly using inherent information on the T-S fuzzy systems to construct a Lyapunov function and properly introducing LMI relaxations will lead to less conservative criteria. All the aforementioned observations motivate us to carry out this study. We propose a novel nonquadratic membership-dependent Lyapunov functions, which can be numerically solved for a given $\mathrm{T}-\mathrm{S}$ fuzzy system to reduce the conservativeness compared with existing stability criteria.

In this paper, the problem of stability of T-S fuzzy systems in continuous-time domain will be investigated. The main contributions of this paper lie in that the so-called nonquadratic membership-dependent Lyapunov function, which is formulated in a high degree form of both the system states and the normalized membership functions, is proposed giving rise to less conservative and numerically easily verified stability criteria for T-S fuzzy systems. Moreover, it is also shown that the conservativeness of our conditions can be reduced if the degree of Lyapunov function increases. The rest of the paper is organized as follows. Section II recalls some definitions with respect to T-S fuzzy systems and reviews some useful existing works. In Section III, the existence conditions for the nonquadratic membership-dependent Lyapunov function are presented to ensure the stability of the underlying T-S fuzzy systems, and the relationships among these conditions are also discussed. Two numerical examples are given in Section IV to demonstrate the validity and less conservativeness of the obtained results. Finally, conclusions are presented in Section V.

Notations: In this paper, the notations used are fairly standard. $A \succ 0$ and $x \succ 0$ (or $A \succeq 0, x \succeq 0$ ) mean that all elements of matrix $A$ and $x$ are positive (or nonnegative); the notation $P>$ $0(\geq 0)$ means that $P$ is a real-symmetric and positive-definite (semipositive definite) matrix; $\mathbb{R}, \mathbb{R}^{n}$, and $\mathbb{R}^{n \times n}$ denote the field of real numbers, $n$-dimensional Euclidean space, and the space of $n \times n$ matrices with real entries, respectively; $\mathbb{N}_{+}$stands for the set of positive integers; $x^{\{m\}}$ is a base vector that contains all homogeneous monomials of degree $m$ in $x ; I_{n}$ is the identity matrix of order $n ; \lambda(A)$ are the eigenvalues of $A ; A \equiv\left[a_{i j}\right]_{n \times n}$, where $a_{i j}$ is the $i$ th line and $j$ th column entry of $A$; for a given vector $v, v_{i}$ (or $\left.(v)_{i}\right)$ stands for its $i$ th entry; $\vartheta(n, m) \equiv$ $(n+m-1) ! /((n-1) ! m !) ; v(x, m, y, n)$ represents a polynomial of degree $m$ and $n$ in $x$ and $y$, respectively, $\forall m, n \in \mathbb{Z}_{+}$; for $h \in \mathbb{R}^{n},\|h\|_{\infty}=\max \left\{\left|h_{1}\right|,\left|h_{2}\right|, \ldots,\left|h_{n}\right|\right\} ; A \otimes B$ refers to the Kronecker product of matrices $A$ and $B$; and $x^{\otimes m}=$ $\overbrace{x \otimes x \otimes \ldots \otimes x}$ denotes the $m$ th Kronecker power in $x$. In addition, the notation $\operatorname{co}\left\{h^{1}, h^{2}, \ldots h^{m}\right\}$ denotes the convex hull of $h^{1}, h^{2}, \ldots, h^{m}$

\section{PROBLEM FORMULATION AND PRELIMINARIES}

The model that is proposed in [37] is described by fuzzy rules, which expresses local linear relations of a nonlinear system, and can be represented as follows:

$$
\begin{aligned}
& \text { Model rule } i: \text { IF } \theta_{1}(t) \text { is } M_{1}^{i} \text { and } \cdots \theta_{p}(t) \text { is } M_{p}^{i} \\
& \text { THEN } \dot{x}(t)=A_{i} x(t), i \in R=\{1,2, \ldots r\}
\end{aligned}
$$

where $x(t) \in \mathbb{R}^{n}$ is the state vector; $M_{j}^{i}$ is the fuzzy set; $r$ denotes the number of model rules; $\theta(t)=\left[\theta_{1}(t)\right.$, $\left.\theta_{2}(t), \ldots, \theta_{p}(t)\right]^{T}$ is the premise variables vector, and each $\theta_{j}(t)$ is a measurable time-varying quantity that may be states, measurable external variables, and/or time; and $A_{i}, i \in R$, are real matrices with appropriate dimensions. By using a standard fuzzy inference method, that is, using a singleton fuzzier, and center-average defuzzier, a more compact presentation of the continuous-time T-S fuzzy model can be given by

$$
\dot{x}(t)=A(h(t)) x(t)=\sum_{i=1}^{r} h_{i}(\theta(t)) A_{i} x(t)
$$

where $h_{i}(\theta(t))$ are the normalized membership functions

$$
h_{i}(\theta(t))=\frac{\Pi_{j=1}^{p} M_{j}^{i}\left(\theta_{j}(t)\right)}{\sum_{i=1}^{r} \Pi_{j=1}^{p} M_{j}^{i}\left(\theta_{j}(t)\right)}
$$

with $M_{j}^{i}\left(\theta_{j}(t)\right)$ representing the grade of membership of premise variable $\theta_{j}(t)$ in $M_{j}^{i}$. In this paper, it is assumed 
that the normalized membership function satisfies the following properties:

$$
\begin{aligned}
h(\theta(t))= & {\left[h_{1}(\theta(t)), h_{2}(\theta(t)), \ldots, h_{r}(\theta(t))\right]^{T} } \\
\in & \left\{h(\theta(t)) \in \mathbb{R}^{r}: \sum_{i=1}^{r} h_{i}(t)=1, h_{i}(t) \geq 0\right\} \\
& \left|\dot{h}_{i}(\theta(t))\right| \leq \phi_{i}, i \in R .
\end{aligned}
$$

Therefore, $\dot{h}(\theta(t)) \in \operatorname{co}\left\{h^{1}, h^{2}, \ldots h^{l}\right\}$, where $h^{j} \in \mathbb{R}^{r}, j \in$ $\{1,2, \ldots, l\}$ are given vertices with $\left\|h^{j}\right\|_{\infty} \leq \max _{i} \phi_{i}$, with $\sum_{i=1}^{r} h_{i}^{j}=0$. For simplicity, in what follows, $h(\theta(t))$ will be denoted by $h(t)$.

In this paper, on the basis of Lyapunov stability theory, we aim to develop less conservative stability conditions for T-S fuzzy system (2) by exploring a new Lyapunov function. Before proceeding, the following necessary definitions and lemmas are given, which will be used to develop our main results in this paper.

Definition 1 [8]: Let $\dot{x}(t)=A x(t)$, where $A$ is an $n \times n$ matrix. Then, $A_{\{m\}}$ is a $\vartheta(n, m) \times \vartheta(n, m)$ matrix that satisfies the following equation:

$$
\dot{x}^{\{m\}}(t)=A_{\{m\}} x^{\{m\}}(t)=\frac{\partial x^{\{m\}}(t)}{\partial x} A x .
$$

We extend Definition 1 for system (2) in the following.

Definition 2: Considering system (2), we define that $A_{\{m\}}(h(t))$ is a $\vartheta(n, m) \times \vartheta(n, m)$ matrix that satisfies the following equation:

$$
\dot{x}^{\{m\}}(t)=A_{\{m\}}(h(t)) x^{\{m\}}(t)=\frac{\partial x^{\{m\}}(t)}{\partial x} A(h(t)) x .
$$

Definition 3 [33]: If $\operatorname{co}\left\{x_{1}, x_{2}, \ldots, x_{m}\right\}$ denotes the convex hull of $x_{1}, x_{2}, \ldots, x_{m}$, then $\operatorname{co}\left\{x_{1}, x_{2}, \ldots, x_{m}\right\}=\left\{\sum_{i=1}^{m}\right.$ $\left.\alpha_{i} x_{i}: \alpha_{i} \geq 0,1 \leq i \leq m, \sum_{i=1}^{m} \alpha_{i}=1\right\}$.

Lemma 1 [7]: Let $K_{m} \in \mathbb{R}^{n^{m} \times \vartheta(n, m)}$ be the matrix that satisfies $x^{\otimes m}(t)=K_{m} x^{\{m\}}(t)$. Then, the matrix $A_{\{m\}}$ in (5) can be given by

$$
A_{\{m\}}=\left(K_{m}^{T} K_{m}\right)^{-1} K_{m}^{T}\left(\sum_{i=0}^{m-1} I_{n^{m-1-i}} \otimes A \otimes I_{n^{i}}\right) K_{m} .
$$

Lemma 2: The matrix $A_{\{m\}}(h(t))$ in (6) can be given by

$$
\begin{aligned}
A_{\{m\}}(h(t))= & \sum_{i=1}^{r} h_{i}(t)\left[\left(K_{m}^{T} K_{m}\right)^{-1} K_{m}^{T}\right. \\
& \left.\times\left(\sum_{i=0}^{m-1} I_{n^{m-1-i}} \otimes A_{i} \otimes I_{n^{i}}\right) K_{m}\right] .
\end{aligned}
$$

Proof: From Definition 1, it can be seen that $A_{\{m\}}$ depends linearly on $A$. Therefore, we can get (8) from Definition 2 and Lemma 1.

Lemma 3: Let $A \in \mathbb{R}^{n_{1} \times n_{2}}$ and $B \in \mathbb{R}^{n_{2} \times n_{3}} ;$ then, $(A B)^{\otimes k}$ $=A^{\otimes k} B^{\otimes k}$.
Lemma 4: For given matrices $A \in \mathbb{R}^{n_{1} \times n_{1}}$ and $B \in \mathbb{R}^{n_{2} \times n_{2}}$, if $\lambda(A)=\left\{\epsilon_{1}, \epsilon_{2}, \ldots \epsilon_{n_{1}}\right\}$ and $\lambda(B)=\left\{\varepsilon_{1}, \varepsilon_{2}, \ldots \varepsilon_{n_{2}}\right\}$, then $\lambda(A \otimes B)=\left\{\epsilon_{i} \varepsilon_{j}, i=1, \ldots, n_{1}, j=1, \ldots, n_{2}\right\}$.

Remark 1: Note that Lemmas 3 and 4 can be trivially proved by some properties of Kronecker product [10] and standard matrix manipulations.

\section{MAIN RESULTS}

In this section, the following nonquadratic membershipdependent type of Lyapunov function is proposed to obtain the stability criteria for T-S fuzzy system (2):

$$
v(x(t), 2 m, h(t), z)=\sum_{i} w_{i}(x(t), h(t))
$$

where $i \in\{1,2, \ldots, \vartheta(n, 2 m) \vartheta(r, z)\}$ and $w_{i}(x(t), h(t))$ are homogeneous forms of $x(t)$ and $h(t)$ with order $2 m$ and $z$, respectively. For instance, $\forall x(t), h(t) \in \mathbb{R}^{2}$

$$
\begin{aligned}
& v(x(t), 2, h(t), 1) \\
= & a_{1} h_{1}(t) x_{1}^{2}(t)+a_{2} h_{1}(t) x_{1}(t) x_{2}(t)+a_{3} h_{1}(t) x_{2}^{2}(t) \\
& +a_{4} h_{2}(t) x_{1}^{2}(t)+a_{5} h_{2}(t) x_{1}(t) x_{2}(t)+a_{6} h_{2}(t) x_{2}^{2}(t)
\end{aligned}
$$

where $a_{i}, i \in\{1,2, \ldots, 6\}$, are the real coefficients. Furthermore, we define the degree of the aforementioned Lyapunov function as $(m, z)$. Therefore, based on Lyapunov's direct method, the main objective of this paper is to propose a numerical method to identify $a_{i}$ for system (2), such that along the system solutions

$$
v(x(t), 2 m, h(t), z)>0 \forall x(t) \in \mathbb{R}^{n} / 0^{n}, h(t) \text { satisfying (3) }
$$

$\dot{v}(x(t), 2 m, h(t), z)<0 \forall x(t) \in \mathbb{R}^{n} / 0^{n}, h(t)$ satisfying (3).

Remark 2: By nonquadratic polynomial Lyapunov function to analyzing the stability of T-S fuzzy system, see [12] and [40], where the Lyapunov functions are formulated in a high-order form of system state $x(t)$ for a class of polynomial fuzzy system. However, different from [40], the one in (9) is constructed by not only $x(t)$, but also $h(t)$ in their high orders. From a mathematical point of view, the Lyapunov function in (9), taking full advantage of the inherent information on the system, has the potential to improve the stability criteria, which will be discussed in more detail later on.

Now, based on the new type of Lyapunov function proposed previously, we are in a position to present our main results in this paper.

Theorem 1: Consider the T-S fuzzy system (2), if there exist parameter vectors

$$
\begin{aligned}
\eta & \in \mathbb{R}^{\vartheta(n, m) \vartheta(r, z)(\vartheta(n, m) \vartheta(r, z)+1) / 2-\vartheta(n, 2 m) \vartheta(r, 2 z)} \\
\lambda_{j} & \in \mathbb{R}^{\vartheta(n, m) \vartheta(r, z+1)(\vartheta(n, m) \vartheta(r, z+1)+1) / 2-\vartheta(n, 2 m) \vartheta(r, 2 z+2)} \\
j & \in\{1,2, \cdots, l\}
\end{aligned}
$$

such that, $\forall j \in\{1,2, \ldots, l\}$,

$$
\begin{aligned}
V+V(\eta) & >0 \\
X_{j}\left(\eta, h^{j}\right)+X_{j}\left(\eta, h^{j}, \lambda_{j}\right) & <0
\end{aligned}
$$


where

$$
\begin{aligned}
V+V(\eta) & \in \mathbb{R}^{\vartheta(n, m) \vartheta(r, z) \times \vartheta(n, m) \vartheta(r, z)} \\
X_{j}\left(\eta, h^{j}\right)+X_{j}\left(\eta, h^{j}, \lambda_{j}\right) & \in \mathbb{R}^{\vartheta(n, m) \vartheta(r, z+1) \times \vartheta(n, m) \vartheta(r, z+1)}
\end{aligned}
$$

are linear parameterization expressions of the matrices that represent a polynomial $v(x(t), 2 m, h(t), z)$ and its time derivative in $h^{j}$, respectively, then there exists a nonquadratic type of Lyapunov function $v(x(t), 2 m, h(t), z)$, which guarantees that system (2) is asymptotically stable.

Proof: A sufficient condition for (10) is the existence of appropriately defined SOS [31], [40] decompositions in $x(t)$, which can be verified by the existence of a positive-definite matrix $\mathcal{V}(h(t))$ (often called the Gram matrix [31]) for any $h(t)$, such that

$$
v(x(t), 2 m, h(t), z)=\left(x^{\{m\}}(t)\right)^{T} \mathcal{V}(h(t)) x^{\{m\}}(t)
$$

where $\mathcal{V}(h(t))$ is a matrix whose entries are homogeneous forms of $h(t)$ with degree $z$. Then, by choosing a matrix $V$ appropriately, we can rewrite (14) as

$$
\begin{aligned}
& v(x(t), 2 m, h(t), z) \\
= & \left(x^{\{m\}}(t)\right)^{T}\left(\sqrt{h(t)}^{\{z\}} \otimes I_{\vartheta(n, m)}\right)^{T} V \\
& \times\left(\sqrt{h(t)}^{\{z\}} \otimes I_{\vartheta(n, m)}\right) x^{\{m\}}(t) .
\end{aligned}
$$

It is noted that the representation of matrix $V$ describing $v(x(t), 2 m, h(t), z)$ is not unique. Let $V$ be a symmetric matrix with variable entries; then, for a given polynomial $v(x(t), 2 m, h(t), z)$, by expanding the right-hand side of (15) and matching the corresponding coefficients, we can get linear constraints on the entries of $V$ in a linear system, where the number of independent constraints is $\vartheta(n, 2 m) \vartheta(r, 2 z)$. Therefore, a linear parameterization expression for the family of matrix $V$ representing $v(x(t), 2 m, h(t), z)$ is obtained in the following:

$$
V_{1}(\eta)=V+V(\eta)
$$

where $\eta \in \mathbb{R}^{\vartheta(n, m) \vartheta(r, z)(\vartheta(n, m) \vartheta(r, z)+1) / 2-\vartheta(n, 2 m) \vartheta(r, 2 z)}$ is a vector of free parameters. It follows that

$$
\begin{aligned}
& v(x(t), 2 m, h(t), z) \\
= & \left(x^{\{m\}}(t)\right)^{T}\left(\sqrt{h(t)}\{z\} \otimes I_{\vartheta(n, m)}\right)^{T} V_{1}(\eta) \\
& \times\left(\sqrt{h(t)}{ }^{\{z\}} \otimes I_{\vartheta(n, m)}\right) x^{\{m\}}(t) .
\end{aligned}
$$

It is, therefore, obvious that if (12) holds, $v(x(t), 2 m, h(t), z)>$ $0 \forall x(t) \in \mathbb{R}^{n}$ and $\forall h(t)$ [defined in (3)].

On the other hand, (17) can be rewritten as follows:

$$
\begin{aligned}
& v(x(t), 2 m, h(t), z) \\
= & \left(x^{\{m\}}(t)\right)^{T}\left(\sqrt{h(t)}{ }^{\{z\}} \otimes I_{\vartheta(n, m)}\right)^{T} V_{1}(\eta) \\
& \left.\times(\sqrt{h(t)})^{\{z\}} \otimes I_{\vartheta(n, m)}\right) x^{\{m\}}(t) \\
= & \left(x^{\{m\}}(t)\right)^{T} V_{1}(\eta, h(t)) x^{\{m\}}(t)
\end{aligned}
$$

$$
\begin{aligned}
= & \left(\sqrt{h(t)}^{\{z\}}\right)^{T}\left(x^{\{m\}}(t) \otimes I_{\vartheta(r, z)}\right)^{T} V_{2}(\eta) \\
& \times\left(x^{\{m\}}(t) \otimes I_{\vartheta(r, z)}\right) \sqrt{h(t)}\{z\} \\
= & \left(\sqrt{h(t)}^{\{z\}}\right)^{T} V_{2}(\eta, x(t)) \sqrt{h(t)}\{z\} .
\end{aligned}
$$

Then, from (3), (4), (18), Definitions 2, 3, and Lemma 2, one has

$$
\begin{aligned}
& \dot{v}(x(t), 2 m, h(t), z) \\
= & \frac{\partial v(x(t), 2 m, h(t), z)}{\partial x^{\{m\}}} \frac{d x^{\{m\}}}{d t} \\
& +\frac{\partial v(x(t), 2 m, h(t), z)}{\partial \sqrt{h(t)}} \frac{d z\}}{d \sqrt{h(t)}\{z\}} \\
= & 2\left(x^{\{m\}}(t)\right)^{T} V_{1}(\eta, h(t)) A_{\{m\}}(h(t)) x^{\{m\}}(t) \\
& +2\left(\sqrt{h(t)^{\{z\}}}\right)^{T} V_{2}(\eta, x(t)) Y(h(t)) \dot{h}(t) \\
= & 2 \sum_{j=1}^{l}\left[\alpha_{j}(t)\left(x^{\{m\}}(t)\right)^{T} V_{1}(\eta, h(t))\left(K_{m}^{T} K_{m}\right)^{-1} K_{m}^{T}\right. \\
& \times\left(\sum_{i=0}^{m-1} I_{n^{m-1-i}} \otimes \sum_{i=1}^{r} h_{i}(t) A_{i} \otimes I_{n^{i}}\right) K_{m} x^{\{m\}}(t) \\
& \left.+\left(\sum_{i=1}^{r} h_{i}(t)\right)^{2}(\sqrt{h(t)}\{z\})^{T} V_{2}(\eta, x(t)) Y(h(t)) \alpha_{j}(t) h^{j}\right] \\
= & 2 \sum_{j=1}^{l} \alpha_{j}(t)\left[v_{1}(\eta)+v_{2}\left(\eta, h^{j}\right)\right]
\end{aligned}
$$

where

$$
\begin{gathered}
\sum_{j=1}^{l} \alpha_{j}(t)=1, \alpha_{j}(t) \geq 0 \quad \forall t \geq 0 \\
v_{1}(\eta)=\left(x^{\{m\}}(t)\right)^{T} V_{1}(\eta, h(t))\left(K_{m}^{T} K_{m}\right)^{-1} K_{m}^{T} \\
\\
\times\left(\sum_{i=0}^{m-1} I_{n^{m-1-i}} \otimes \sum_{i=1}^{r} h_{i}(t) A_{i} \otimes I_{n^{i}}\right) K_{m} x^{\{m\}}(t) \\
v_{2}\left(\eta, h^{j}\right)=\left(\sum_{i=1}^{r} h_{i}(t)\right)^{2}\left(\sqrt{h(t)}^{\{z\}}\right)^{T} V_{2}(\eta, x(t)) Y(h(t)) h^{j}
\end{gathered}
$$

and $Y(h(t)) \in \mathbb{R}^{\vartheta(r, z) \times r}$ is a polynomial matrix in $h(t)$ whose $(i, j)$ th entry is given by

$$
Y_{i j}(h(t))=\frac{\partial\left(\sqrt{h(t)}^{\{z\}}\right)_{i}}{\partial h_{j}(t)} .
$$

It is noted that both $v_{1}(\eta)$ and $v_{2}\left(\eta, h^{j}\right)$ are homogeneous forms of degree $2 m$ in $x(t)$ and degree $z+1$ in $h(t)$. Thus, one can find appropriate matrices $X_{j}\left(\eta, h^{j}\right)$ $\in \mathbb{R}^{\vartheta(n, m) \vartheta(r, z+1) \times \vartheta(n, m) \vartheta(r, z+1)}$, such that, $\forall j \in\{1,2$, 
$\ldots, l\}$,

$$
\begin{aligned}
& v_{1}(\eta)+v_{2}\left(\eta, h^{j}\right) \\
= & \left(x^{\{m\}}(t)\right)^{T}\left(\sqrt{h(t)}\{z+1\} \otimes I_{\vartheta(n, m)}\right)^{T} X_{j}\left(\eta, h^{j}\right) \\
& \times\left(\sqrt{h(t)}{ }^{\{z+1\}} \otimes I_{\vartheta(n, m)}\right) x^{\{m\}}(t) .
\end{aligned}
$$

Analogous to the procedure of (14)-(17), we can also get a linear parameterization $X_{j}\left(\lambda_{j}\right)=X_{j}\left(\eta, h^{j}\right)+X_{j}\left(\eta, h^{j}, \lambda_{j}\right)$ for $X_{j}\left(\eta, h^{j}\right)$ describing $v_{1}(\eta)+v_{2}\left(\eta, h^{j}\right), \forall j \in\{1,2, \cdots, l\}$, such that

$$
\begin{aligned}
& v_{1}(\eta)+v_{2}\left(\eta, h^{j}\right) \\
= & \left(x^{\{m\}}(t)\right)^{T}\left(\sqrt{h(t)}^{\{z+1\}} \otimes I_{\vartheta(n, m)}\right)^{T} X_{j}\left(\lambda_{j}\right) \\
& \times\left(\sqrt{h(t)}{ }^{\{z+1\}} \otimes I_{\vartheta(n, m)}\right) x^{\{m\}}(t)
\end{aligned}
$$

where $\lambda_{j} \in \mathbb{R}^{\frac{\vartheta(n, m) \vartheta(r, z+1)(\vartheta(n, m) \vartheta(r, z+1)+1)}{2}-\vartheta(n, 2 m) \vartheta(r, 2 z+2)}$ is a vector of free parameters. Equations (19)-(21) imply that (13) is sufficient for (11).

Finally, we conclude that if (12) and (13) hold, there exists a Lyapunov function for system (2) in the form of (9) to ensure its stability, and the corresponding Lyapunov function of degree $(m, z)$ can be obtained by (17). This completes the proof.

Remark 3: A sufficient condition for the existence of nonquadratic membership-dependent Lyapunov function (9) is obtained in Theorem 1. It can be seen from the proof that the set of feasible solutions is the intersections of two affine subspaces (confined by the Lyapunov function and its time derivative, respectively) and two cones (confined by the corresponding positive-definite and negative-definite matrices, respectively).

Remark 4: It should be mentioned that a significant feature of the considered Lyapunov function is the higher order compositions of system state $x(t)$ and normalized membership function $h(t)$, compared with most existing ones in the literature, which enables us to take more freedom into the LMIs formulated in (12)-(13) with the growth of the degree of the Lyapunov function (9). However, a question naturally arises: Can the conservativeness of the stability conditions in Theorem 1 be reduced when the degree grows? The following Theorem gives an answer to this question.

Theorem 2: For given scalars $m, z \in \mathbb{N}_{+}$, if there exist parameter vectors

$\eta \in \mathbb{R}^{\vartheta(n, m) \vartheta(r, z)(\vartheta(n, m) \vartheta(r, z)+1) / 2-\vartheta(n, 2 m) \vartheta(r, 2 z)}$

$\lambda_{j} \in \mathbb{R}^{\vartheta(n, m) \vartheta(r, z+1)(\vartheta(n, m) \vartheta(r, z+1)+1) / 2-\vartheta(n, 2 m) \vartheta(r, 2 z+2)}$

$$
j \in\{1,2, \ldots, l\}
$$

such that (12) and (13) are satisfied, then for any scalar $k \in \mathbb{N}_{+}$, there exist parameter vectors

$$
\begin{aligned}
\tilde{\eta} & \in \mathbb{R}^{\vartheta(n, k m) \vartheta(r, k z)(\vartheta(n, k m) \vartheta(r, k z)+1) / 2-\vartheta(n, k m) \vartheta(r, 2 k z)} \\
\tilde{\lambda}_{j} & \in \mathbb{R}^{\frac{\vartheta(n, k m) \vartheta(r, k z+1)(\vartheta(n, k m) \vartheta(r, k z+1)+1)}{2}-\vartheta(n, k m) \vartheta(r, 2 k z+2)}
\end{aligned}
$$

such that, $\forall j \in\{1,2, \cdots, l\}$,

$$
\tilde{V}+\tilde{V}(\tilde{\eta})>0
$$

$$
\tilde{X}_{j}\left(\tilde{\eta}, h^{j}\right)+\tilde{X}_{j}\left(\tilde{\eta}, h^{j}, \tilde{\lambda}_{j}\right)<0
$$

where

$$
\begin{aligned}
\tilde{V}+\tilde{V}(\tilde{\eta}) & \in \mathbb{R}^{\vartheta(n, k m) \vartheta(r, k z) \times \vartheta(n, k m) \vartheta(r, k z)} \\
\tilde{X}_{j}\left(\tilde{\eta}, h^{j}\right)+\tilde{X}_{j}\left(\tilde{\eta}, h^{j}, \tilde{\lambda}_{j}\right) & \in \mathbb{R}^{\vartheta(n, k m) \vartheta(r, k z+1) \times \vartheta(n, k m) \vartheta(r, k z+1)}
\end{aligned}
$$

are linear parameterization expressions of the matrices representing a polynomial $\tilde{v}(x(t), 2 k m, h(t), k z)$ and its time derivative in $h^{j}$, respectively. Moreover, if (22) and (23) have a solution, $\tilde{v}(x(t), 2 k m, h(t), k z)$ is a Lyapunov function of degree $(k m, k z)$ ensuring the stability of the T-S fuzzy system (2).

Proof: If (12) and (13) hold, it follows that

$$
\begin{aligned}
& v(x(t), 2 m, h(t), z) \\
= & \left(x^{\{m\}}(t)\right)^{T}\left(\sqrt{h(t)}\{z\} \otimes I_{\vartheta(n, m)}\right)^{T} V_{1}(\eta) \\
& \times\left(\sqrt{h(t)}^{\{z\}} \otimes I_{\vartheta(n, m)}\right) x^{\{m\}}(t) \\
> & 0
\end{aligned}
$$

is a Lyapunov function of degree $(m, z)$ guaranteeing the stability of system (2). Here, we particularly choose

$$
\tilde{v}(x(t), 2 k m, h(t), k z)=v^{k}(x(t), 2 m, h(t), z)
$$

to be a Lyapunov function candidate. Then, Lemma 3 and (24) yield

$$
\begin{aligned}
& \tilde{v}(x(t), 2 k m, h(t), k z) \\
= & v^{k}(x(t), 2 m, h(t), z) \\
= & {\left[\left(x^{\{m\}}(t)\right)^{T}\left(\sqrt{h(t)}{ }^{\{z\}} \otimes I_{\vartheta(n, m)}\right)^{T} V_{1}(\eta)\right.} \\
& \left.\times\left(\sqrt{h(t)}^{\{z\}} \otimes I_{\vartheta(n, m)}\right) x^{\{m\}}(t)\right]^{\otimes k} \\
= & {\left[\left(x^{\{m\}}(t)\right)^{T}\left(\sqrt{h(t)}^{\{z\}} \otimes I_{\vartheta(n, m)}\right)^{T}\right]^{\otimes k} V_{1}^{\otimes k}(\eta) } \\
& \times\left[\left(\sqrt{h(t)}^{\{z\}} \otimes I_{\vartheta(n, m)}\right) x^{\{m\}}(t)\right]^{\otimes k} \\
= & \left(x^{\{k m\}}(t)\right)^{T}(\sqrt{h(t)}\{k z\} \\
& \times\left(\sqrt{h(t)}^{\{k z\}} \otimes I_{\vartheta(n, k m)}\right) x^{\{k m\}}(t) \\
= & \left(x^{\{k m\}}(t)\right)^{T}\left(\sqrt{h(t)} \tilde{V}_{1}\right. \\
& \times\left(\sqrt{h(t)}^{\{k z\}} \otimes I_{\vartheta(n, k m)}\right) x^{\{k m\}}(t) \\
> & 0
\end{aligned}
$$

where $\tilde{V}_{1}=T_{1}^{T} V_{1}^{\otimes k}(\eta) T_{1}$ with full rank matrix $T_{1}$ satisfying

$$
\begin{aligned}
& {\left[\left(\sqrt{h(t)}^{\{z\}} \otimes I_{\vartheta(n, m)}\right) x^{\{m\}}(t)\right]^{\otimes k} } \\
= & T_{1}\left(\sqrt{h(t)}^{\{k z\}} \otimes I_{\vartheta(n, k m)}\right) x^{\{k m\}}(t) .
\end{aligned}
$$


It is noted that $\lambda\left(V_{1}(\eta)\right)>0$. Thus, by Lemma 4 , one can get $\lambda\left(V_{1}^{\otimes k}(\eta)\right)>0$, which implies that $\tilde{V}_{1}>0$, i.e., there exists a vector $\tilde{\eta}$ such that $(22)$ is satisfied.

On the other hand, followed by (19), (21), (24), Definition 3, and Lemma 3, one can get

$$
\begin{aligned}
& \dot{\tilde{v}}(x(t), 2 k m, h(t), k z) \\
& =\frac{d v^{k}(x(t), 2 m, h(t), z)}{d t} \\
& =k v^{k-1}(x(t), 2 m, h(t), z) \dot{v}(x(t), 2 m, h(t), z) \\
& =k\left[\left(x^{\{m\}}(t)\right)^{T}\left(\sqrt{h(t)}^{\{z\}} \otimes I_{\vartheta(n, m)}\right)^{T} V_{1}(\eta)\right. \\
& \left.\times\left(\sqrt{h(t)}^{\{z\}} \otimes I_{\vartheta(n, m)}\right) x^{\{m\}}(t)\right]^{\otimes(k-1)} \\
& \otimes 2 \sum_{j=1}^{l} \alpha_{j}(t)\left[\left(x^{\{m\}}(t)\right)^{T}\left(\sqrt{h(t)}^{\{z+1\}} \otimes I_{\vartheta(n, m)}\right)^{T}\right. \\
& \left.X_{j}\left(\lambda_{j}\right)\left(\sqrt{h(t)}^{\{z+1\}} \otimes I_{\vartheta(n, m)}\right) x^{\{m\}}(t)\right] \\
& =2 \sum_{j=1}^{l} k \alpha_{j}(t)\left\{\left(x^{\{k m\}}(t)\right)^{T}\left(\sqrt{h(t)}^{\{k z+1\}} \otimes I_{\vartheta(n, k m)}\right)^{T}\right. \\
& T_{2}^{T}\left[V_{1}^{\otimes(k-1)}(\eta) \otimes X_{j}\left(\lambda_{j}\right)\right] T_{2}\left(\sqrt{h(t)}^{\{k z+1\}}\right. \\
& \left.\left.\otimes I_{\vartheta(n, k m)}\right) x^{\{k m\}}(t)\right\} \\
& =\sum_{j=1}^{l} \alpha_{j}(t)\left\{\left(x^{\{k m\}}(t)\right)^{T}\left(\sqrt{h(t)}^{\{k z+1\}} \otimes I_{\vartheta(n, k m)}\right)^{T}\right. \\
& \times\left[\tilde{X}_{j}\left(\tilde{\eta}, h^{j}\right)+\tilde{X}_{j}\left(\tilde{\eta}, h^{j}, \tilde{\lambda}_{j}\right)\right]\left(\sqrt{h(t)}^{\{k z+1\}}\right. \\
& \left.\left.\otimes I_{\vartheta(n, k m)}\right) x^{\{k m\}}(t)\right\}
\end{aligned}
$$

where the full rank matrix $T_{2}$ satisfies

$$
\begin{aligned}
& \left.T_{2}\left(\sqrt{h(t)}^{\{k z+1\}} \otimes I_{\vartheta(n, k m)}\right) x^{\{k m\}}(t)\right\} \\
= & {\left[\left(\sqrt{h(t)}^{\{z\}} \otimes I_{\vartheta(n, m)}\right) x^{\{m\}}(t)\right]^{\otimes(k-1)} \otimes } \\
& \times\left[\left(\sqrt{h(t)}^{\{z+1\}} \otimes I_{\vartheta(n, m)}\right) x^{\{m\}}(t)\right] .
\end{aligned}
$$

By Lemma 4, it is also clear that $T_{2}^{T}\left[V_{1}^{\otimes(k-1)}(\eta) \otimes\right.$ $\left.X_{j}\left(\lambda_{j}\right)\right] T_{2}<0$ since $V_{1}^{\otimes(k-1)}(\eta)>0$ and $X_{j}\left(\lambda_{j}\right)<0$. So $(26)$ shows that if (12) and (13) are satisfied, there exist $\tilde{\eta}$ and $\tilde{\lambda}_{j}$ such that (23) holds.

Finally, it is straightforward to see from (25) and (26) that if (22) and (23) have a solution, then $\tilde{v}(x(t), 2 k m, h(t), k z)$ is a Lyapunov function ensuring the stability of the T-S fuzzy system (2).

Remark 5: It should be noted from Theorem 2 that for a given T-S fuzzy system, if we can find a nonquadratic membershipdependent Lyapunov function of degree $(m, z)$, then we can also find a Lyapunov function of degree $(k m, k z)$ assuring the
TABLE I

RELATIONS BETWEEN THE NUMBERS OF LMI VARIABLES AND DEGREES OF LYAPUNOV FUNCTIONS

\begin{tabular}{c|c|c|c}
\hline \hline $\begin{array}{c}\text { Degree of } \\
\text { Lyapunov function }\end{array}$ & $(m, z)=(1,1)$ & $(m, z)=(2,2)$ & $(m, z)=(3,3)$ \\
\hline $\begin{array}{c}\text { Numbers of } \\
\text { LMI variables }\end{array}$ & 19 & 121 & 409 \\
\hline \hline
\end{tabular}

stability of the underlying system. However, the converse is not true, which implies that increasing the orders of $x(t)$ and $h(t)$ in a Lyapunov function (9) simultaneously will relax the corresponding stability conditions obtained.

Remark 6: It should be pointed out that the reduction of conservativeness is at the expense of increasing computational complexity since more LMI variables will be involved with the growth of the degree. Table I provides the relations between the numbers of LMI variables and degrees of Lyapunov functions for given parameters $n=2, r=2$, and $l=2$. Another problem should also be noted that the system matrices and the parameters of Lyapunov functions in the conditions of Theorem 1 are coupled in a special manner, which is different from some common ones in the literature. This will bring some difficulty in dealing with controller design problems since this coupling is not easily decoupled by some standard LMI techniques used in the literature. Therefore, how to get an equivalent result that decouples the parameters of Lyapunov functions and the system matrices deserves further investigation in future work.

On the other hand, the observations in Remark 5 lead to another question: How do the stability conditions vary with the individual orders of $x(t)$ and $h(t)$ ? This stimulates us to present the following result.

Theorem 3: For any given scalars $m, z \in \mathbb{N}_{+}$, if there exist parameter vectors

$$
\begin{aligned}
\eta & \in \mathbb{R}^{\vartheta(n, m) \vartheta(r, z)(\vartheta(n, m) \vartheta(r, z)+1) / 2-\vartheta(n, 2 m) \vartheta(r, 2 z)} \\
\lambda_{j} & \in \mathbb{R}^{\vartheta(n, m) \vartheta(r, z+1)(\vartheta(n, m) \vartheta(r, z+1)+1) / 2-\vartheta(n, 2 m) \vartheta(r, 2 z+2)} \\
j & \in\{1,2, \ldots, l\}
\end{aligned}
$$

such that (12) and (13) are satisfied, then there exist parameter vectors

$$
\begin{aligned}
\bar{\eta} & \in \mathbb{R}^{\vartheta(n, m) \vartheta(r, z+1)(\vartheta(n, m) \vartheta(r, z+1)+1) / 2-\vartheta(n, 2 m) \vartheta(r, 2 z+2)} \\
\bar{\lambda}_{j} & \in \mathbb{R}^{\vartheta(n, m) \vartheta(r, z+2)(\vartheta(n, m) \vartheta(r, z+2)+1) / 2-\vartheta(n, 2 m) \vartheta(r, 2 z+4)}
\end{aligned}
$$

such that $\forall j \in\{1,2, \ldots, l\}$

$$
\begin{array}{r}
\bar{V}+\bar{V}(\bar{\eta})>0 \\
\bar{X}_{j}\left(\bar{\eta}, h^{j}\right)+\bar{X}_{j}\left(\bar{\eta}, h^{j}, \bar{\lambda}_{j}\right)<0
\end{array}
$$

where

$$
\begin{aligned}
\bar{V}+\bar{V}(\bar{\eta}) & \in \mathbb{R}^{\vartheta(n, m) \vartheta(r, z+1) \times \vartheta(n, m) \vartheta(r, z+1)} \\
\bar{X}_{j}\left(\bar{\eta}, h^{j}\right)+\bar{X}_{j}\left(\bar{\eta}, h^{j}, \bar{\lambda}_{j}\right) & \in \mathbb{R}^{\vartheta(n, m) \vartheta(r, z+2) \times \vartheta(n, m) \vartheta(r, z+2)}
\end{aligned}
$$

are linear parameterization expressions of the matrices representing a polynomial $\bar{v}(x(t), 2 m, h(t), z+1)$ and its time derivative in $h^{j}$, respectively. Moreover, if (27) and (28) have 
a solution, $\bar{v}(x(t), 2 m, h(t), z+1)$ is a Lyapunov function of degree $(m, z+1)$ ensuring the stability of the T-S fuzzy system (2).

Proof: As a special case, we can choose

$$
\bar{v}(x(t), 2 m, h(t), z+1)=\left(\sum_{i=1}^{r} h_{i}(t)\right) v(x(t), 2 m, h(t), z)
$$

to be a Lyapunov function candidate, where $v(x(t), 2 m, h(t), z)$ is a Lyapunov function satisfying (12) and (13). Then, we have that

$$
\begin{aligned}
& \bar{v}(x(t), 2 m, h(t), z+1) \\
& =\left(\sum_{i=1}^{r} h_{i}(t)\right) v(x(t), 2 m, h(t), z) \\
& =\sqrt{h(t)}^{T} \sqrt{h(t)}\left(x^{\{m\}}(t)\right)^{T}\left(\sqrt{h(t)}^{\{z\}} \otimes I_{\vartheta(n, m)}\right)^{T} \\
& V_{1}(\eta)\left(\sqrt{h(t)}^{\{z\}} \otimes I_{\vartheta(n, m)}\right) x^{\{m\}}(t) \\
& =\left(x^{\{m\}}(t)\right)^{T}\left\{\left[\sqrt{h(t)}^{T} I_{r} \sqrt{h(t)}\right] \otimes\right. \\
& \left.\times\left[\left(\sqrt{h(t)}^{\{z\}} \otimes I_{\vartheta(n, m)}\right)^{T} V_{1}(\eta)\left(\sqrt{h(t)}^{\{z\}} \otimes I_{\vartheta(n, m)}\right)\right]\right\} \\
& x^{\{m\}}(t) \\
& =\left(x^{\{m\}}(t)\right)^{T}\left(\sqrt{h(t)} \otimes \sqrt{h(t)}^{\{z\}} \otimes I_{\vartheta(n, m)}\right)^{T} \\
& \times\left[I_{r} \otimes V_{1}(\eta)\right]\left(\sqrt{h(t)} \otimes \sqrt{h(t)}^{\{z\}} \otimes I_{\vartheta(n, m)}\right) x^{\{m\}}(t) \\
& =\left(x^{\{m\}}(t)\right)^{T}\left(\sqrt{h(t)}^{\{z+1\}} \otimes I_{\vartheta(n, m)}\right)^{T}\left(T_{3} \otimes I_{\vartheta(n, m)}\right)^{T} \\
& \times\left[I_{r} \otimes V_{1}(\eta)\right]\left(T_{3} \otimes I_{\vartheta(n, m)}\right)\left(\sqrt{h(t)}^{\{z+1\}} \otimes I_{\vartheta(n, m)}\right) \\
& x^{\{m\}}(t) \\
& =\left(x^{\{m\}}(t)\right)^{T}\left(\sqrt{h(t)}^{\{z+1\}} \otimes I_{\vartheta(n, m)}\right)^{T}(\bar{V}+\bar{V}(\bar{\eta})) \\
& \times\left(\sqrt{h(t)}^{\{z+1\}} \otimes I_{\vartheta(n, m)}\right) x^{\{m\}}(t)
\end{aligned}
$$

where $T_{3}$ is a full rank matrix satisfying $\sqrt{h(t)} \otimes \sqrt{h(t)}\{z\}=$ $T_{3} \sqrt{h(t)}^{\{z+1\}}$. In accordance with the similar development in Theorem 2, it can be seen from (29) that there exists a vector $\bar{\eta}$ such that (27) is satisfied.

On the other hand

$$
\begin{aligned}
& \dot{\bar{v}}(x(t), 2 m, h(t), z+1) \\
= & \left(\sum_{i=1}^{r} \dot{h}_{i}(t)\right) v(x(t), 2 m, h(t), z) \\
& +\dot{v}(x(t), 2 m, h(t), z) \sum_{i=1}^{r} h_{i}(t)
\end{aligned}
$$

$$
\begin{aligned}
& =2 \sum_{j=1}^{l} \alpha_{j}\left[\left(x^{\{m\}}(t)\right)^{T}\left(\sqrt{h(t)}^{\{z+1\}} \otimes I_{\vartheta(n, m)}\right)^{T} X_{j}\left(\lambda_{j}\right)\right. \\
& \left.\times\left(\sqrt{h(t)}^{\{z+1\}} \otimes I_{\vartheta(n, m)}\right) x^{\{m\}}(t)\right] \sqrt{h(t)}^{T} \sqrt{h(t)} \\
& =2 \sum_{j=1}^{l} \alpha_{j}(t)\left[\left(x^{\{m\}}(t)\right)^{T}\left(\sqrt{h(t)}^{T} I_{r} \sqrt{h(t)}\right)\right. \\
& \times\left(\sqrt{h(t)}^{\{z+1\}} \otimes I_{\vartheta(n, m)}\right)^{T} X_{j}\left(\lambda_{j}\right) \\
& \left.\times\left(\sqrt{h(t)}^{\{z+1\}} \otimes I_{\vartheta(n, m)}\right) x^{\{m\}}(t)\right] \\
& =2 \sum_{j=1}^{l} \alpha_{j}(t)\left[( x ^ { \{ m \} } ( t ) ) ^ { T } \left(\sqrt{h(t)} \otimes \sqrt{h(t)}^{\{z+1\}}\right.\right. \\
& \left.\otimes I_{\vartheta(n, m)}\right)^{T}\left(I_{r} \otimes X_{j}\left(\lambda_{j}\right)\right)(\sqrt{h(t)} \otimes \\
& \left.\left.\sqrt{h(t)}^{\{z+1\}} \otimes I_{\vartheta(n, m)}\right) x^{\{m\}}(t)\right] \\
& =2 \sum_{j=1}^{l} \alpha_{j}(t)\left[\left(x^{\{m\}}(t)\right)^{T}\left(\sqrt{h(t)}^{\{z+2\}} \otimes I_{\vartheta(n, m)}\right)^{T}\right. \\
& \times\left(T_{4} \otimes I_{\vartheta(n, m)}\right)^{T}\left(I_{r} \otimes X_{j}\left(\lambda_{j}\right)\right)\left(T_{3} \otimes I_{\vartheta(n, m)}\right) \\
& \left.\times\left(\sqrt{h(t)}^{\{z+2\}} \otimes I_{\vartheta(n, m)}\right) x^{\{m\}}(t)\right] \\
& =\sum_{j=1}^{l} \alpha_{j}(t)\left[\left(x^{\{m\}}(t)\right)^{T}\left(\sqrt{h(t)}^{\{z+2\}} \otimes I_{\vartheta(n, m)}\right)^{T}\right. \\
& \times\left(\bar{X}_{j}\left(\bar{\eta}, h^{j}\right)+\bar{X}_{j}\left(\bar{\eta}, h^{j}, \bar{\lambda}_{j}\right)\right) \\
& \left.\times\left(\sqrt{h(t)}^{\{z+2\}} \otimes I_{\vartheta(n, m)}\right) x^{\{m\}}(t)\right]
\end{aligned}
$$

where $T_{4}$ is a full rank matrix that satisfies $\sqrt{h(t)} \otimes$ $\sqrt{h(t)}^{\{z+1\}}=T_{4} \sqrt{h(t)}^{\{z+2\}}$. Therefore, there exist $\bar{\eta}$ and $\bar{\lambda}_{j}$ satisfying (27) and (28) if $X_{j}\left(\lambda_{j}\right)<0$.

Finally, from (29) and (30), it suffices that if (27) and (28) are feasible, then $\bar{v}(x(t), 2 m, h(t), z+1)$ is a Lyapunov function ensuring the stability of the T-S fuzzy system (2), which completes the proof.

Remark 7: Note that for any given order $m$ of $x(t)$ in a Lyapunov function (9), Theorem 3 indicates that raising individually the $h(t)$ 's order in (9) will accordingly improve the performance of the stability conditions. This highlights, again, the importance of the membership functions in stability analysis for T-S fuzzy systems in the sense of Lyapunov stability, which is indeed the reason why we formulate the Lyapunov function in the form of (9).

Remark 8: We can conclude from Remarks 5 and 7 that the stability conditions will be relaxed by either increasing both orders of $x(t)$ and $h(t)$, or increasing the order of only $h(t)$. Here, a quite meaningful question should be pointed out. For any fixed order of $h(t)$, can the conservativeness of corresponding stability criteria be reduced by formulating higher degree Lyapunov 
functions in $x(t)$ individually? Such a relation cannot be established theoretically and an example will demonstrate this point in the next section.

As two extreme cases, if the degrees of the Lyapunov function (9) are set as $(1,0)$ and $(1,1)$, then the Lyapunov function (9) are reduced to the common quadratic Lyapunov function and fuzzy Lyapunov function, which can be equivalently expressed as

$$
v(x(t), 2, h(t), 0)=x^{T}(t) V x(t)
$$

and

$$
v(x(t), 2, h(t), 1)=x^{T}(t) \sum_{i=1}^{r} h_{i}(t) V_{i} x(t)
$$

respectively. Then, based on these two types of Lyapunov functions, it is not hard to get the following two corollaries, respectively.

Corollary 1: If there exists a symmetric matrix $V \in \mathbb{R}^{n \times n}$, such that, $\forall i \in R$,

$$
\begin{aligned}
V & >0 \\
A_{i}^{T} V+V A_{i} & <0
\end{aligned}
$$

then there exists a common quadratic Lyapunov function $v(x(t), 2, h(t), 0)=x^{T}(t) V x(t)$, which guarantees that system (2) is asymptotically stable.

Remark 9: Based on the Lyapunov function (31), the proof of Corollary 1 can be trivially obtained by Theorem 1 . It is clear that Corollary 1 is identical to [16, Proposition 1].

Corollary 2: If there exist symmetric matrices $V_{i} \in \mathbb{R}^{n \times n}, i \in$ $R$, such that, $\forall i \in\{1,2, \ldots l\},\left(k, k^{\prime}\right) \in R \times R, k \leq k^{\prime}$

$V_{k}>0$

$\sum_{k=1}^{r} h_{k}^{i} V_{k}+\frac{1}{2}\left(A_{k}^{T} V_{k^{\prime}}+V_{k^{\prime}} A_{k}+A_{k^{\prime}}^{T} V_{k}+V_{k} A_{k^{\prime}}\right)<0$

then there exists a fuzzy Lyapunov function $v(x(t), 2, h(t), 1)=$ $x^{T}(t) \sum_{i=1}^{r} h_{i}(t) V_{i} x(t)$, which guarantees that system (2) is asymptotically stable.

Proof: Analogous to the proof of Theorem 1 and choosing $\eta=$ 0 and $\lambda_{j}=0$, we can obtain that if (33) and (34) are satisfied, then

$$
\begin{aligned}
& v(x(t), 2, h(t), 1)=x^{T}(t) \sum_{i=1}^{r} h_{i}(t) V_{i} x(t)>0 \\
& \dot{v}(x(t), 2, h(t), 1) \\
& =\sum_{k=1}^{r} \dot{x}^{T}(t) h_{k}(t) V_{k} x(t)+\sum_{k=1}^{r} x^{T}(t) h_{k}(t) V_{k} \dot{x}(t) \\
& \quad+2 \sqrt{h(t)}^{T} \operatorname{diag}\left\{x^{T}(t) V_{1} x(t), x^{T}(t) V_{2} x(t), \ldots,\right. \\
& \left.x^{T}(t) V_{r} x(t)\right\} \operatorname{diag}\left\{\frac{1}{\sqrt{h_{1}(t)}}, \ldots, \frac{1}{\sqrt{h_{r}(t)}}\right\} \frac{\dot{h}(t)}{2} \\
& =\sum_{k=1}^{r} \sum_{k^{\prime}=1}^{r} h_{k}(t) h_{k^{\prime}}(t) x^{T}(t)\left(A_{k^{\prime}}^{T} V_{k}+V_{k} A_{k^{\prime}}\right) x(t)
\end{aligned}
$$

$$
\begin{aligned}
& +\sum_{i=1}^{l} \alpha_{i}(t)\left[x^{T}(t) V_{1} x(t), \ldots, x^{T}(t) V_{r} x(t)\right] h^{i} \\
= & \frac{1}{2} \sum_{k=1}^{r} \sum_{k^{\prime}=1}^{r} h_{k}(t) h_{k^{\prime}}(t) x^{T}(t)\left(A_{k}^{T} V_{k^{\prime}}+V_{k^{\prime}} A_{k}\right. \\
& \left.+A_{k^{\prime}}^{T} V_{k}+V_{k} A_{k^{\prime}}\right) x(t)+\sum_{i=1}^{l} \sum_{k=1}^{r} \alpha_{i}(t) h_{k}^{i} x^{T}(t) V_{k} x(t) \\
= & \sum_{k=1}^{r} \sum_{k^{\prime}=1}^{r} \sum_{i=1}^{l} h_{k}(t) h_{k^{\prime}}(t) \alpha_{i}(t) x^{T}(t) \\
& \left(\sum_{k=1}^{r} h_{k}^{i} V_{k}+\frac{A_{k}^{T} V_{k^{\prime}}+V_{k^{\prime}} A_{k}+A_{k^{\prime}}^{T} V_{k}+V_{k} A_{k^{\prime}}}{2}\right) x(t) \\
& <0 .
\end{aligned}
$$

These indicate that if (33) and (34) are feasible, then $v(x(t), 2, h(t), 1)=x^{T}(t) \sum_{i=1}^{r} h_{i}(t) V_{i} x(t)$ is a fuzzy Lyapunov function ensuring the asymptotic stability of the system (2), which completes the proof.

Remark 10: One can get from $\left\|h^{j}\right\|_{\infty} \leq \max _{i} \phi_{i}$, and (34) that for any symmetric matrices $V_{k}>0, k \in R$

$$
\begin{aligned}
& \sum_{k=1}^{r} h_{k}^{i} V_{k}+\frac{1}{2}\left(A_{k}^{T} V_{k^{\prime}}+V_{k^{\prime}} A_{k}+A_{k^{\prime}}^{T} V_{k}+V_{k} A_{k^{\prime}}\right) \\
\leq & \sum_{k=1}^{r} \phi_{k} V_{k}+\frac{1}{2}\left(A_{k}^{T} V_{k^{\prime}}+V_{k^{\prime}} A_{k}+A_{k^{\prime}}^{T} V_{k}+V_{k} A_{k^{\prime}}\right) .
\end{aligned}
$$

Therefore, we can conclude from the aforementioned inequality that the conditions of [38, Th. 1] are sufficient for the criteria of Corollary 2, i.e., if there exist symmetric matrices $V_{k}>0, k \in$ $R$ such that the conditions of [38, Th. 1] are feasible, then (33) and (34) are feasible as well, but not vice versa.

\section{NUMERICAL EXAMPLE}

We provide the following numerical examples in this section to verify our main results that are developed in this paper.

Example 1: Consider the system used in [5] and [25], which has three rules and the following local matrices:

$$
\begin{array}{ll}
A_{1} & =\left[\begin{array}{cc}
0.00 & 1.00 \\
-0.06 & -1.00
\end{array}\right], \quad A_{2}=\left[\begin{array}{cc}
0.00 & a \\
-1.94 & -1.00
\end{array}\right] \\
A_{3} & =\left[\begin{array}{cc}
0.00 & b \\
-0.50 & -1.50
\end{array}\right] .
\end{array}
$$

The normalized membership functions are as follows:

$$
\begin{aligned}
& h_{1}\left(x_{1}(t)\right)=\frac{\omega_{1}\left(x_{1}(t)\right)}{\omega_{1}\left(x_{1}(t)\right)+\omega_{2}\left(x_{1}(t)\right)+\omega_{2}\left(x_{1}(t)\right)} \\
& h_{2}\left(x_{1}(t)\right)=\frac{\omega_{2}\left(x_{1}(t)\right)}{\omega_{1}\left(x_{1}(t)\right)+\omega_{2}\left(x_{1}(t)\right)+\omega_{2}\left(x_{1}(t)\right)} \\
& h_{3}\left(x_{1}(t)\right)=\frac{\omega_{3}\left(x_{1}(t)\right)}{\omega_{1}\left(x_{1}(t)\right)+\omega_{2}\left(x_{1}(t)\right)+\omega_{2}\left(x_{1}(t)\right)}
\end{aligned}
$$



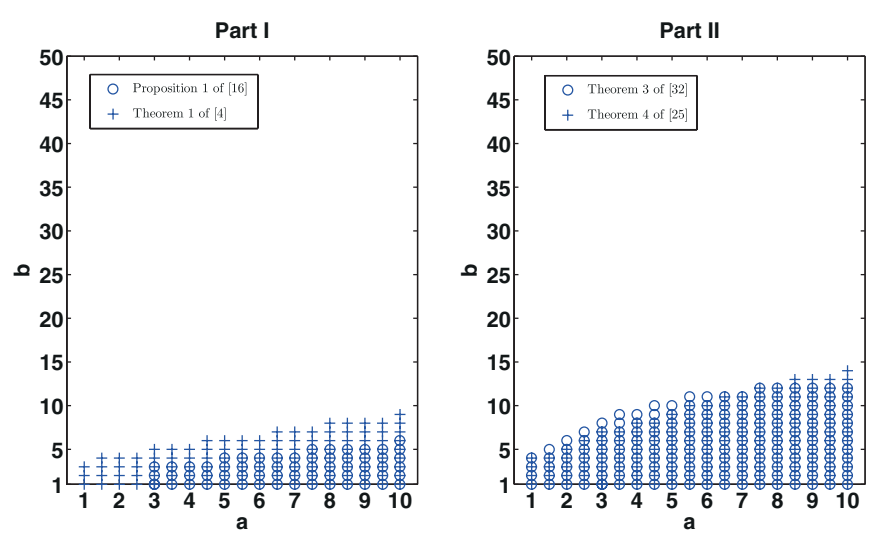

Fig. 1. Stability regions obtained by some membership-independent conditions recently reported.
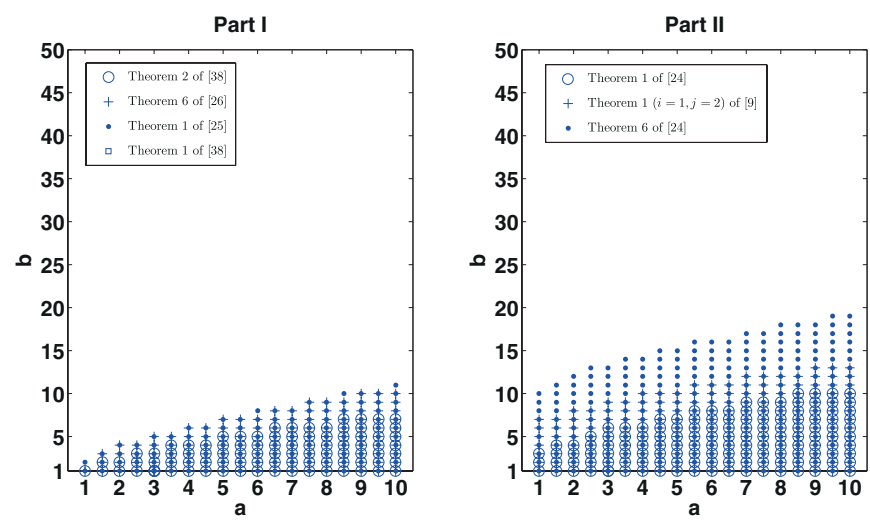

Fig. 2. Stability regions obtained by some membership-dependent conditions recently reported.

where

$$
\begin{aligned}
& \omega_{1}\left(x_{1}(t)\right)=\exp \left\{-\frac{1}{2}\left(\frac{x_{1}(t)+5}{2}\right)^{2}\right\} \\
& \omega_{2}\left(x_{1}(t)\right)=\exp \left\{-\frac{1}{2}\left(\frac{x_{1}(t)}{2}\right)^{2}\right\} \\
& \omega_{3}\left(x_{1}(t)\right)=\exp \left\{-\frac{1}{2}\left(\frac{x_{1}(t)-5}{2}\right)^{2}\right\} .
\end{aligned}
$$

Next, for $a \in[1,10], b \in[1,50]$, and $\left|\dot{h}_{i}(t)\right| \leq 0.3, \forall i \in$ $\{1,2,3\}$, we will compare the stability regions ${ }^{1}$ obtained by our approach and the ones obtained by other typical existing methods in the literature [4], [9], [16], [24], [25], [26], [32], [38]. The comparison results are depicted in Figs. 1, 2, and 3, by which the following conclusions can be drawn.

1) It can be seen from Fig. 2(Part I) that, $\forall(a, b) \in[1,10] \times$ $[1,50]$, we cannot find a feasible solution (marked by “ $\square$ ”) using [38, Th. 1], which means that we cannot conclude whether the system is stable or not.

2) From Figs. 1, 2, and 3, we can see that when the degree of the Lyapunov function candidate is not less than $(1,2)$,

\footnotetext{
${ }^{1}$ Here the "stability regions" is slightly abused to mean the achieved scope of $a$ and $b$ such that the system is stable.
}
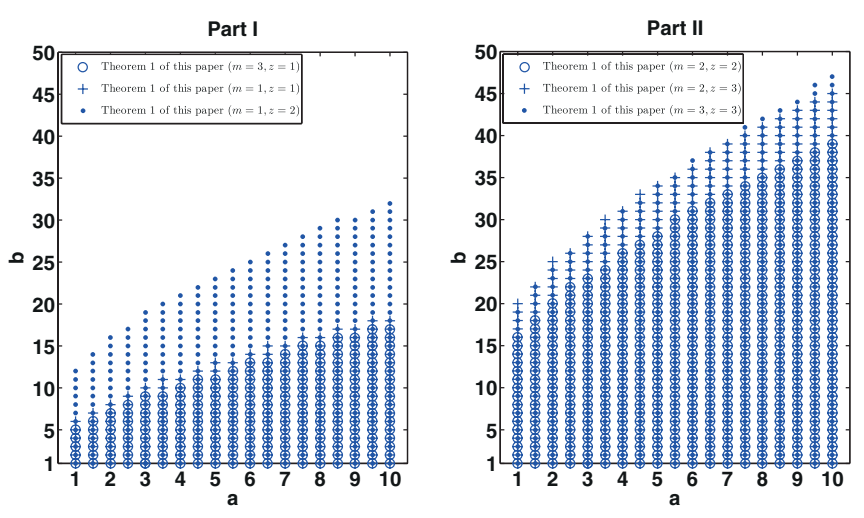

Fig. 3. Stability regions obtained by Theorem 1 of this paper with different degrees of Lyapunov functions.

TABLE II

STABILITY REGION BY INDIVIDUALLY INCREASING THE DEGREE OF $x(t)$ IN LYAPUNOV FUNCTION CANDIDATE

\begin{tabular}{c|c}
\hline \hline $\begin{array}{c}\text { Variations of degree of the } \\
\text { Lyapunov function candidate }\end{array}$ & $\begin{array}{c}\text { Between the different degrees } \\
\text { of } m \text { and } z\end{array}$ \\
\hline$(m=1, z=2) \rightarrow(m=2, z=2)$ & Increased \\
\hline$(m=1, z=1) \rightarrow(m=3, z=1)$ & Decreased \\
\hline$(m=2, z=3) \rightarrow(m=3, z=3)$ & Uncertain \\
\hline \hline
\end{tabular}

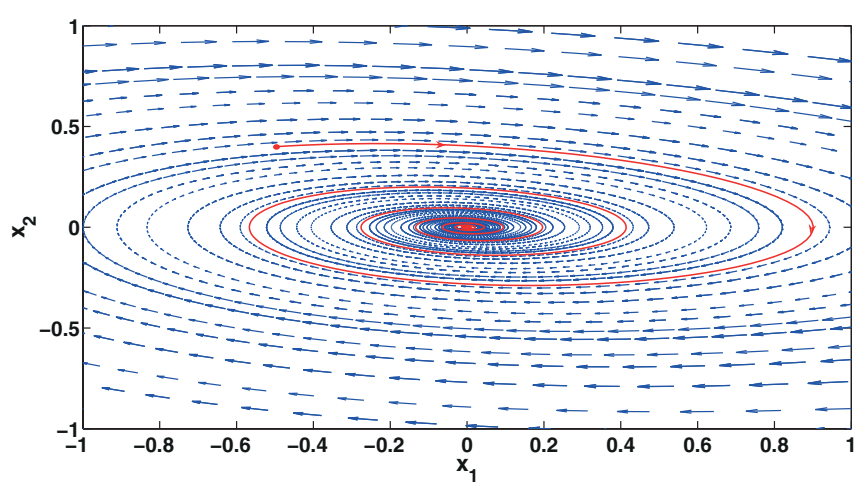

Fig. 4. Phase portrait of the underlying system for given parameters $a=$ $10, b=40$.

Theorem 1 in our paper can yield larger stability regions than most existing methods.

3) It is shown in Fig. 3 that the stability region obtained in Theorem 1 can be enlarged by simultaneously increasing the degrees of $x(t)$ and $h(t)$ in the Lyapunov function candidate (see $(1,1),(2,2)$, and $(3,3)$, for example), which verifies Theorem 2 .

4) It can be easily seen from Fig. 3 that the stability region obtained in Theorem 1 can be expanded by raising the degree of $h(t)$ in the Lyapunov function candidate (see $(1,1),(1,2)$ or $(2,2),(2,3)$ or $(3,1),(3,3)$, for example), which verifies Theorem 3 .

5) As we have mentioned in Remark 8, it can be also seen from Fig. 3 and Table II that there is no certain rule on the monotonicity in the variations of the stability regions as the degree of $x(t)$ varies.

For given parameters $a=10$ and $b=40$ (the corresponding system is stable, which cannot be testified out by existing 
TABLE III

COMPARISON RESUlts of THE MAXIMAL VALUE OF $k$ FOR THE SiXTh-ORDER POLYNOMIAL LyaPUNOV FunCtions IN $x(t)$

\begin{tabular}{c|c|c|c|c|c}
\hline \hline Method & Theorem 1 in [40] & Theorem 1 in [12] & $\begin{array}{c}\text { Theorem 1 } \\
(m=3, z=1)\end{array}$ & $\begin{array}{c}\text { Theorem 1 } \\
(m=3, z=2)\end{array}$ & $\begin{array}{c}\text { Theorem 1 } \\
(m=3, z=3)\end{array}$ \\
\hline$k_{\max }$ & 6.17 & \multirow{2}{*}{6.30} & $\begin{array}{c}\phi_{i}=1: 10.52 \\
\phi_{i}=5: 6.46 \\
\phi_{i}=10: 6.28\end{array}$ & $\begin{array}{c}\phi_{i}=1: 16.64 \\
\phi_{i}=5: 6.90 \\
\phi_{i}=10: 6.41\end{array}$ & $\begin{array}{c}\phi_{i}=1: 18.18 \\
\phi_{i}=5: 7.16 \\
\phi_{i}=10: 6.85\end{array}$ \\
\hline \hline
\end{tabular}

methods other than Theorem 1 in our paper), the phase portrait of the underlying system is depicted in Fig. 4, where the red curve is the state trajectory with $x(0)=[-0.5,0.4]$.

Example 2: Let us consider system (2) with two rules and the following local matrices [12], [19], [40]:

$$
A_{1}=\left[\begin{array}{cc}
0 & 1 \\
-2 & -1
\end{array}\right], \quad A_{2}=\left[\begin{array}{cc}
0 & 1 \\
-2-k & -1
\end{array}\right] \text {. }
$$

Our purpose here is to compare our results with those obtained by a polynomial Lyapunov function approach (but only in system state $x(t)$ ). As in [19], the system is analyzed by the computation of $k_{\max }$ for different $\phi_{i}, i \in R$, such that the stability is guaranteed for any $0 \leq k \leq k_{\max }$. The comparison results are listed in Table III for the sixth-order polynomial Lyapunov functions in $x(t)$. We get from Table III that the conservativeness can be further reduced by increasing the degree of $h(t)$.

\section{CONCLUSION}

The stability problem for T-S fuzzy systems has been studied in this paper. As a first attempt, a class of nonquadratic membership-dependent Lyapunov functions for the underlying systems has been proposed, where the Lyapunov function candidate is formulated in polynomial forms in both the state $x(t)$ and the normalized membership function $h(t)$. Then, the membership-dependent conditions are developed for the existence of this new type of Lyapunov function for T-S fuzzy systems, such that the system is stable. The results are further verified to have less conservativeness with increased degrees of the Lyapunov function. Furthermore, it has been demonstrated that the stability criterion can be relaxed by individually increasing the order of $h(t)$ for a fixed order of $x(t)$ in the Lyapunov function. Numerical examples are provided to verify the advantages of the proposed approach, by comparisons with some existing results.

\section{ACKNOWLEDGMENT}

The authors would like to thank the associate editor and reviewers for their very constructive comments and suggestions, which have helped greatly improve the quality and presentation of this paper.

\section{REFERENCES}

[1] W. Assawinchaichote and S. K. Nguang, "Fuzzy $H_{\infty}$ output feedback control design for singularly perturbed systems with pole placement constraints: An LMI approach," IEEE Trans. Fuzzy Syst., vol. 14, no. 3, pp. 361-371, Jun. 2006.

[2] A. Benzaouia and A. E. Hajjaji, "Delay-dependent stabilization conditions of controlled positive T-S fuzzy systems with time varying delay," Int. J. Innovative Comput., Inf. Control, vol. 7, no. 4, pp. 1533-1548, 2011.

[3] M. Bernal, T. Guerra, and A. Kruszewski, "A membership-functiondependent approach for stability analysis and controller synthesis of
Takagi-Sugenomodels," Fuzzy Sets Syst., vol. 160, no. 19, pp. 2776-2795, 2009.

[4] M. Chadli, D. Maquin, and J. Ragot, "Relaxed stability conditions for Takagi-Sugeno fuzzy systems," in Proc. IEEE Int. Conf. Syst., Man, Cybern., Nashville, TN, USA, 2000, pp. 3514-3519.

[5] M. Chadli, D. Maquin, and J. Ragot, "Nonquadratic stability analysis of Takagi-Sugeno models," in Proc. 41th IEEE Conf. Control Decis., Las Vegas, NV, USA, Dec. 2002, pp. 2143-2148.

[6] W. J. Chang, C. C. Ku, and P. H. Huang, "Robust fuzzy control via observer feedback for passive stochastic fuzzy systems with time-delay and multiplicative noise," Int. J. Innovative Comput. Inf. Control, vol. 7, no. 1, pp. 345-364, 2011.

[7] G. Chesi, A. Garulli, A. Tesi, and A. Vicino, "Homogeneous Lyapunov functions for systems with structured uncertainties," Automatica, vol. 39, no. 6, pp. 1027-1035, 2003.

[8] G. Chesi, A. Garulli, A. Tesi, and A. Vicino, Homogeneous polynomial forms for robustness analysis of uncertain systems homogeneous polynomial forms for robustness analysis of uncertain system. (Lecture Notes in Control and Information Sciences). Berlin, Germany: Springer, 2009.

[9] F. Faria, G. Silva, V. Oliveira, and R. Cardim, "Improving the stability conditions of T-S fuzzy systems with fuzzy Lyapunov functions,"in Proc. 18th IFAC World Cong., Milano, Italy, 2011, pp. 10881-10886.

[10] A. Graham, Kronecker Products and Matrix Calculus with Applications. Chichester, U.K.: Ellis Horwood, 1981.

[11] K. Guelton, T. M. Guerra, M. Bernal, T. Bouarar, and N. Manamanni, "Comments on fuzzy control systems design via fuzzy Lyapunov functions," IEEE Trans. Syst., Man Cybern. B, vol. 40, no. 3, pp. 970-972, Jun. 2010.

[12] K. Guelton, N. Manamanni, D. L. Koumba-Emianiwe, and C. D. Chinh, "SOS stability conditions for nonlinear systems based on a polynomial fuzzy Lyapunov function," in Proc. 18th IFAC World Cong., Milano, Italy, 2011, pp. 12777-12782.

[13] C. P. Huang, "Model based fuzzy control with affine T-S delayed models applied to nonlinear systems," Int. J. Innovative Comput. Inf. Control, vol. 8, no. 5, pp. 2979-2993, 2012.

[14] B. Jiang, Z. Mao, and P. Shi, " $H_{\infty}$-filter design for a class of networked control systems via T-S fuzzy-model approach," IEEE Trans. Fuzzy Syst., vol. 18 , no. 1, pp. 201-208, Feb. 2010

[15] B. Jiang, K. Zhang, and P. Shi, "Integrated fault estimation and accommodation design for discrete-time Takagi-Sugeno fuzzy systems with actuator faults," IEEE Trans. Fuzzy Syst., vol. 19, no. 2, pp. 291-304, Apr. 2011.

[16] M. Johansson, A. Rantzer, and K. Arzen, "Piecewise quadratic stability of fuzzy systems," IEEE Trans. Fuzzy Syst., vol. 7, no. 6, pp. 713-722, Dec. 1999.

[17] H. R. Karimi and H. Gao, "New delay-dependent exponential $H_{\infty}$ synchronization for uncertain neural networks with mixed time delays," IEEE Trans. Syst., Man Cybern. B, vol. 40, no. 1, pp. 173-185, Feb. 2010.

[18] D. H. Lee, J. B. Park, and Y. H. Joo, "A fuzzy Lyapunov function approach to estimating the domain of attraction for continuous-time Takagi-Sugeno fuzzy systems," Inf. Sci., vol. 185, no. 1, pp. 230-248, 2011.

[19] D. H. Lee, J. B. Park, and Y. H. Joo, "A new fuzzy Lyapunov function for relaxed stability condition of continuous-time Takagi-Sugeno fuzzy systems," IEEE Trans. Fuzzy Syst., vol. 19, no. 4, pp. 785-791, Aug. 2011.

[20] K. Y. Lian, H. W. Tu, and J. J. Liou, "Stability conditions for LMI-based fuzzy control from viewpoint of membership functions," IEEE Trans. Fuzzy Syst., vol. 14, no. 6, pp. 874-884, Dec. 2006.

[21] J. Liang, Z. Wang, and X. Liu, "On passivity and passification of stochastic fuzzy systems with delays: The discrete-time case," IEEE Trans. Syst., Man Cybern. B, vol. 40, no. 3, pp. 964-969, Jun. 2010.

[22] C. M. Lin, "Supervisory recurrent fuzzy neural network control of wing rock for slender delta wings," IEEE Trans. Fuzzy Syst., vol. 12, no. 5, pp. 733-742, Oct. 2004.

[23] H. Liu, F. Sun, and Z. Sun, "Stability analysis and synthesis of fuzzy singularly perturbed systems," IEEE Trans. Fuzzy Syst., vol. 13, no. 2, pp. 273-284, Apr. 2005. 
[24] L. Mozelli and R. Palhares, "Stability analysis of Takagi-Sugeno fuzzy systems via LMI: Methodologies based on a new fuzzy Lyapunov function," Revista Controle Automacao, vol. 22, no. 6, pp. 664-676, 2011.

[25] L. Mozelli, R. Palhares, and G. Avellar, "A systematic approach to improve multiple Lyapunov function stability and stabilization conditions for fuzzy systems," Inf. Sci., vol. 179, no. 8, pp. 1149-1162, 2009.

[26] L. Mozelli, R. Palhares, F. Souza, and E. Mendes, "Reducing conservativeness in recent stability conditions of T-S fuzzy systems," Automatica, vol. 45, no. 6, pp. 1580-1583, 2009.

[27] L. Mozelli, F. Souza, and R. Palhares, "New Lyapunov function and extra information on membership functions for improving stability conditions of TS systems," in Proc. 18th IFAC World Cong., Milano, Italy, 2011, pp. 3398-3402.

[28] S. K. Nguang, W. Assawinchaichotea, and P. Shi, "Robust $H_{\infty}$ control design for fuzzy singularly perturbed systems with Markovian jumps: An LMI approach," IET Control Theory Appl., vol. 1, no. 4, pp. 893-908, 2007.

[29] S. K. Nguang, P. Shi, and S. Ding, "Fault detection for uncertain fuzzy systems: An LMI approach," IEEE Trans. Fuzzy Syst., vol. 15, no. 6, pp. 1251-1262, Dec. 2007.

[30] J. T. Pan, T. M. Guerra, S. M. Fei, and A. Jaadari, "Nonquadratic stabilization of continuous T-S fuzzy models: LMI solution for a local approach," IEEE Trans. Fuzzy Syst., vol. 20, no. 3, pp. 594-602, Jun. 2012.

[31] V. Powers and T. Wormann, "An algorithm for sums of squares of real polynomials," J. Pure Appl. Algebra, vol. 127, no. 1, pp. 99-104, 1998.

[32] B. Rhee and S. Won, "A new fuzzy Lyapunov function approach for a Takagi-Sugeno fuzzy control system design," Fuzzy Sets Syst., vol. 157, pp. 1211-1228, 2006.

[33] R. Rockafellar, Convex Analysis. Princeton, NJ, USA, Princeton Press, 1970.

[34] A. Sala and C. Arino, "Relaxed stability and performance conditions for Takagi-Sugeno fuzzy systems with knowledge on membership function overlap," IEEE Trans. Syst., Man Cybern. B, vol. 37, no. 3, pp. 727-732, Jun. 2007.

[35] M. Sugeno, "Fuzzy control: Principles, practice, and perspectives," in Proc. IEEE Int. Conf. Fuzzy Syst., San Diego, CA, USA, 1992, pp. 109114.

[36] C. H. Sun, S. W. Lin, and Y. T. Wang, "Relaxed stabilization conditions for switching T-S fuzzy systems with practical constraints," Int. J. Innovative Comput., Inf. Control, vol. 8, no. 6, pp. 4133-4145, 2012.

[37] T. Takagi and M. Sugeno, "Fuzzy identification of systems and its applications to modeling and control," IEEE Trans. Syst., Man Cybern., vol. 15 , no. 1, pp. 116-132, Jan./Feb. 1985.

[38] K. Tanaka, T. Hori, and H. O. Wang, "A multiple Lyapunov function approach to stabilization of fuzzy control systems," IEEE Trans. Fuzzy Syst., vol. 11, no. 4, pp. 582-589, Aug. 2003.

[39] K. Tanaka, H. Ohtake, and H. O. Wang, "Guaranteed cost control of polynomial fuzzy systems via a sum of squares approach," IEEE Trans. Syst., Man, Cybern. B, vol. 39, no. 2, pp. 561-567, Apr. 2009.

[40] K. Tanaka, H. Yoshida, H. Ohtake, and H. O. Wang, "A sum-of-squares approach to modeling and control of nonlinear dynamical systems with polynomial fuzzy systems," IEEE Trans. Fuzzy Syst., vol. 17, no. 4, pp. 911-922, Aug. 2009.

[41] M. C. M. Teixeira, E. Assuncao, and R. G. Avellar, "On relaxed LMIbased designs for fuzzy regulators and fuzzy observers," IEEE Trans. Fuzzy Syst., vol. 11, no. 5, pp. 613-623, Oct. 2003.

[42] H. O. Wang, K. Tanaka, and M. F. Griffin, "An approach to fuzzy control of nonlinear system: Stability and design issues," IEEE Trans. Fuzzy Syst., vol. 4, no. 1, pp. 14-23, Feb. 1996.

[43] W. Wang and C. Sun, "A relaxed stability criterion for T-S fuzzy discrete systems," IEEE Trans. Syst., Man, Cybern. B, vol. 34, no. 5, pp. 21552158, Oct. 2004.

[44] Z. Wu, P. Shi, H. Su, and J. Chu, "Reliable $H_{\infty}$ control for discrete-time fuzzy systems with infinite-distributed delay," IEEE Trans. Fuzzy Syst., vol. 20, no. 1, pp. 22-31, Feb. 2012.

[45] Y. Xia, H. Yang, P. Shi, and M. Fu, "Constrained infinite-horizon model predictive control for fuzzy-discrete-time systems," IEEE Trans. Fuzzy Syst., vol. 18, no. 2, pp. 429-436, Apr. 2010.

[46] K. Zhang, B. Jiang, and P. Shi, "Fault estimation observer design for discrete-time Takagi-Sugeno fuzzy systems based on piecewise Lyapunov functions," IEEE Trans. Fuzzy Syst., vol. 20, no. 1, pp. 192-200, Feb. 2012.

[47] X. Zhao, L. Zhang, P. Shi, and M. Liu, "Stability and stabilization of switched linear systems with mode-dependent average dwell time," IEEE Trans. Automat. Control, vol. 57, no. 7, pp. 1809-1815, Jul. 2012.

[48] S. Zhou, G. Feng, J. Lam, and S. Xu, " Robust $H_{\infty}$ control for discrete fuzzy systems via basis-dependent Lyapunov functions," Inf. Sci., vol. 174, nos. 3-4, pp. 197-217, 2005. 